\title{
Immuno-PET in Inflammatory Bowel Disease: Imaging CD4-Positive T Cells in a Murine Model of Colitis
}

\author{
Amanda C. Freise ${ }^{1}$, Kirstin A. Zettlitz ${ }^{1}$, Felix B. Salazar ${ }^{1}$, Richard Tavaré ${ }^{1}$, Wen-Ting K. Tsai ${ }^{1}$, Arion F. Chatziioannou ${ }^{1}$, \\ Nora Rozengurt ${ }^{2}$, Jonathan Braun ${ }^{1,2}$, and Anna M. Wu ${ }^{1}$ \\ ${ }^{I}$ Crump Institute for Molecular Imaging, Department of Molecular and Medical Pharmacology, David Geffen School of Medicine at \\ UCLA, Los Angeles, California; and ${ }^{2}$ Department of Pathology and Laboratory Medicine, David Geffen School of Medicine at UCLA, \\ Los Angeles, California
}

\begin{abstract}
Inflammatory bowel diseases (IBDs) in humans are characterized in part by aberrant CD4-positive (CD4+) T-cell responses. Currently, identification of foci of inflammation within the gut requires invasive procedures such as colonoscopy and biopsy. Molecular imaging with antibody fragment probes could be used to noninvasively monitor cell subsets causing intestinal inflammation. Here, GK1.5 cysdiabody (cDb), an antimouse CD4 antibody fragment derived from the GK1.5 hybridoma, was used as a PET probe for CD4+ T cells in the dextran sulfate sodium (DSS) mouse model of IBD. Methods: The DSS mouse model of IBD was validated by assessing changes in CD4+ T cells in the spleen and mesenteric lymph nodes (MLNs) using flow cytometry. Furthermore, CD4 $+\mathrm{T}$ cell infiltration in the colons of colitic mice was evaluated using immunohistochemistry. ${ }^{89} \mathrm{Zr}$-labeled GK1.5 cDb was used to image distribution of CD4+ $T$ cells in the abdominal region and lymphoid organs of mice with DSS-induced colitis. Region-of-interest analysis was performed on specific regions of the gut to quantify probe uptake. Colons, ceca, and MLNs were removed and imaged ex vivo by PET. Imaging results were confirmed by ex vivo biodistribution analysis. Results: An increased number of CD4+ T cells in the colons of colitic mice was confirmed by anti-CD4 immunohistochemistry. Increased uptake of $89 \mathrm{Zr}$-maleimide-deferoxamine (malDFO)-GK1.5 cDb in the distal colon of colitic mice was visible in vivo in PET scans, and regionof-interest analysis of the distal colon confirmed increased activity in DSS mice. MLNs from colitic mice were enlarged and visible in PET images. Ex vivo scans and biodistribution confirmed higher uptake in DSS-treated colons (DSS, $1.8 \pm 0.40$; control, $0.45 \pm 0.12$ percentage injected dose $[\% \mathrm{ID}]$ per organ, respectively), ceca (DSS, $1.1 \pm 0.38$; control, $0.35 \pm 0.09 \% \mathrm{ID}$ per organ), and MLNs (DSS, $1.1 \pm 0.58$; control, $0.37 \pm 0.25 \%$ ID per organ). Conclusion: ${ }^{89} \mathrm{Zr}$-malDFOGK1.5 cDb detected CD4+ T cells in the colons, ceca, and MLNs of colitic mice and may prove useful for further investigations of CD4+ $T$ cells in preclinical models of IBD, with potential to guide development of antibody-based imaging in human IBD.
\end{abstract}

Key Words: immuno-PET; CD4; inflammatory bowel disease; ${ }^{89} \mathrm{Zr}$; diabody

J Nucl Med 2018; 59:980-985

DOI: $10.2967 /$ jnumed.117.199075

Received Aug. 14, 2017; revision accepted Dec. 24, 2017.

For correspondence or reprints contact: Anna M. Wu, Crump Institute for Molecular Imaging, University of California, Los Angeles, 570 Westwood

Plaza, CNSI 4335, P.O. Box 951770, Los Angeles, CA 90095.

E-mail: awu@mednet.ucla.edu

Guest Editor: Carolyn J. Anderson, University of Pittsburgh

Published online Jan. 11, 2018.

COPYRIGHT (C 2018 by the Society of Nuclear Medicine and Molecular Imaging.
$\mathbf{T}$ he gut is a crucial compartment of the immune system. Lymphocytes reside in multiple locations throughout the gut, including the intestine, gut-associated lymphoid tissue such as Peyer patches and lymphoid follicles, and mesenteric lymph nodes (MLNs) (1). The immune system must balance between tolerating the many food- and microbiome-derived antigens sampled from the gut and mounting an immune response to pathogens. Dysregulation of this delicate balance can lead to inflammatory bowel disease (IBD), a family of disorders characterized in part by abnormal and excessive T-cell responses (2).

CD4-positive (CD4+) T cells are known mediators of inflammation in IBD. Crohn's disease and ulcerative colitis each involves different subsets of CD4+ T cells, including T helper type 1 (Th1), Th2, and Th17 (2). Mouse models of IBD have helped advance understanding of how certain immune cell subsets, including CD4+ T cells, contribute to gut inflammation. Several models, such as the CD4+ CD45RB ${ }^{\text {hi }}$ T-cell transfer model and the 2,4,6-trinitrobenzenesulfonic acid/oxalozone model, are CD4+ T-cell-dependent and are used to study contributions of the adaptive immune system to IBD (3). The dextran sulfate sodium (DSS) model of colitis is often used to investigate innate immune cell contributions, as it is characterized by an initial influx of neutrophils, followed by infiltration of T cells. Although CD4 + T cells are not required for the induction of DSS colitis $(4,5)$, they do increase in number in the colon as the disease progresses (6). Hall et al. (6) found that the peak number of CD4+ T cells occurred on day 12 after initiation of DSS administration. In addition, changes in the percentage of T cells in spleen and MLNs can occur as early as day 1 (6), indicating that during acute colitis the adaptive immune system is already changing, even though $\mathrm{T}$ cells are not required for induction. Given these findings, continued investigation into the role of CD4+ T cells in the DSS colitis model is warranted.

Molecular imaging has been used to assess inflammation in murine models of colitis. ${ }^{18} \mathrm{~F}-\mathrm{FDG}$ and PET imaging were used in the DSS model to detect inflammation at several time points during progression of acute disease (7) and to correlate uptake in the colon with histologic damage and expression of perforin $(8),{ }^{18} \mathrm{~F}$ FDG PET was used to identify inflammation in models with mild or severe chronic colitis, including the $\mathrm{CD}^{+}{ }^{+} \mathrm{CD} 45 \mathrm{RB}^{\text {high }}$ and Gai2 ${ }^{-1-}$ CD3 + T-cell transfer models (9). In humans with IBD, ${ }^{18} \mathrm{~F}-\mathrm{FDG}$ PET can identify inflammation with estimated sensitivity of $85 \%$ and specificity of $87 \%$ (10) and provide information complementary to standard methods such as colonoscopy and clinical symptom tracking (11); one caveat is that the highly variable physiologic uptake of ${ }^{18} \mathrm{~F}$-FDG PET in the gut may reduce its utility as a screening tool (12). 


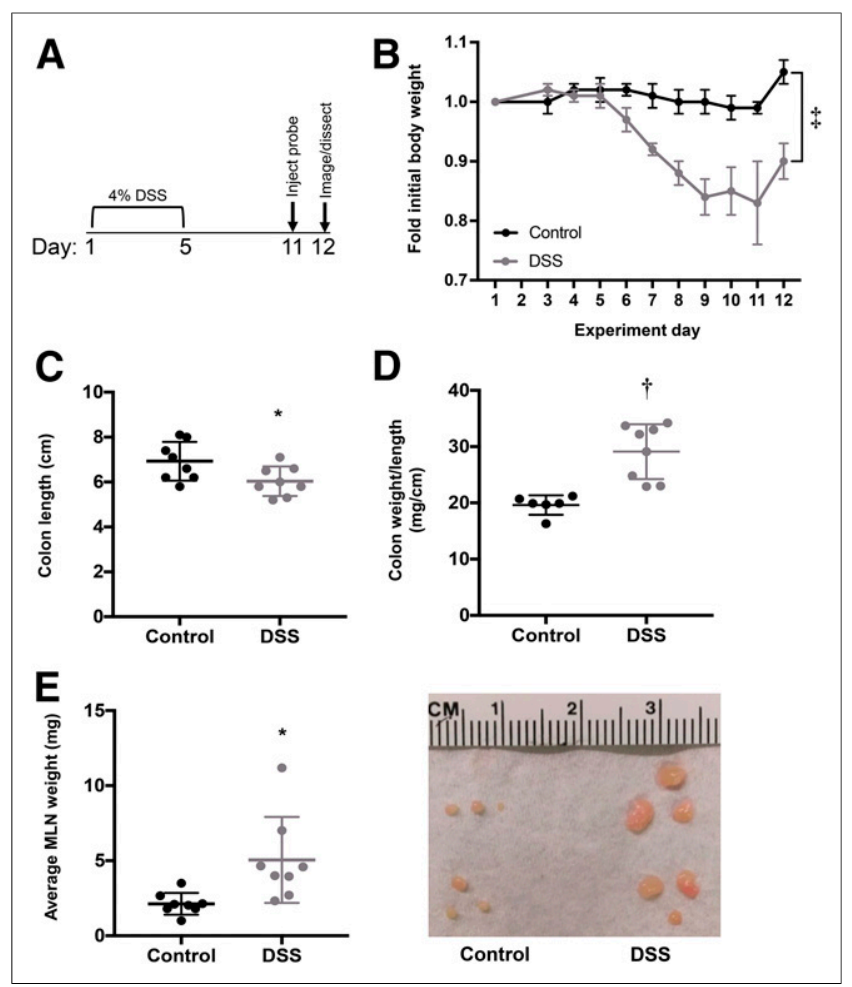

FIGURE 1. Characterization of DSS-induced anatomic changes in colitic mice. (A) Schematic showing days of DSS treatment, injection of ${ }^{89} \mathrm{Zr}$-malDFO-GK1.5 cDb, and PET imaging. (B) Change in body weight during and after DSS treatment $(n=8)$. (C) Colons cut just below cecum, laid flat, and measured ( $n=8$ ). (D) Ratio of colon weight (after removing stool) to length $(n=8)$. (E) MLNs removed, counted, pooled per mouse, and weighed. Each dot represents average weight of $1 \mathrm{MLN}$ from individual mouse ( $n=8$ mice). Image shows 3 representative MLNs from 2 control mice (left) and 2 DSS-treated mice (right). ${ }^{*} P<0.05{ }^{\dagger} P<$ 0.0005. ${ }^{\ddagger} P<0.0001$.

Investigating specific cell subsets involved in IBD requires more specific probes. Molecular imaging with antibodies enables imaging of cell-surface biomarkers (13), and antibodies have been used to image immune cells in models of colitis. Kanwar et al. used a nondepleting intact anti-CD4 antibody for SPECT imaging of CD4+ $\mathrm{T}$ cells in DSS colitis and showed that uptake in the lower abdomen correlated with disease severity and the number of CD4+ T cells present (14). The integrin $\beta_{7}$ subunit, expressed on lymphocytes activated in Peyer patches and MLNs, was recently used as a target for SPECT imaging in DSS-treated colitic mice (15). Dearling et al. developed fragments of the antiintegrin $\beta_{7}$ antibody probe and used them to image lymphocytes in the intestines of colitic mice (16).

We previously described GK1.5 cys-diabody $(\mathrm{cDb})$, an antimouse CD4 antibody fragment, and demonstrated its use in tracking immune system reconstitution posthematopoietic stem cell transplant (17). Subsequent studies investigated the effect of protein dose on image contrast and biologic effect on CD4 $+\mathrm{T}$ cells and showed that low-dose GK1.5 cDb produces high-contrast images of lymphoid organs with minimal effects on CD4+ T-cell function in vivo (18). Here, the utility of anti-CD4 GK1.5 cDb as an immuno-PET probe for regions of CD4+ T-cell infiltration in the murine DSS model of IBD has been evaluated and correlated with ex vivo biodistribution analysis, flow cytometry, and immunohistochemistry of CD4+ T cells in the intestines and lymphoid organs.

\section{MATERIALS AND METHODS}

\section{Animals}

Female C57BL/6 mice 6-12 wk old were obtained from Jackson Laboratories and housed by the Department of Laboratory Animal Medicine at the University of California, Los Angeles, under specific pathogen-free conditions. Animal studies were conducted under protocols approved by the institutional Chancellor's Animal Research Committee.

\section{Induction and Assessment of Colitis}

DSS (40,000 kDa; Sigma) was dissolved in autoclaved tap water, sterile-filtered, and provided ad libitum. On the basis of published literature (6) and dose-finding pilot studies (data not shown), we determined that $4 \%$ DSS administered for $5 \mathrm{~d}$ induced infiltration of mononuclear cells into the colon by day 12 . Regular water was restored on day 6. Mice were weighed every day and examined for signs of colitis, which was evaluated by a disease activity index modified from Sha et al. (19): weight loss $(0$, none; $1,1 \%-4 \% ; 2,5 \%-10 \% ; 3$, $11 \%-20 \%$; 4, >20\%), fecal blood (0, none; 2 , blood present in stool; 4 , gross bleeding from anus), and stool consistency ( 0 , normal; 2, loose stools; 4, diarrhea). On day 12, the mice were euthanized, and the colon, cecum, and MLNs were removed. MLNs were pooled per individual mouse, weighed, and divided by the number of MLNs collected to get the average weight of 1 MLN for each mouse.

\section{GK1.5 cDb Administration and PET/CT Imaging}

Antimouse CD4 GK1.5 cDb was produced, purified, and radiolabeled as described previously $(17,18)$.

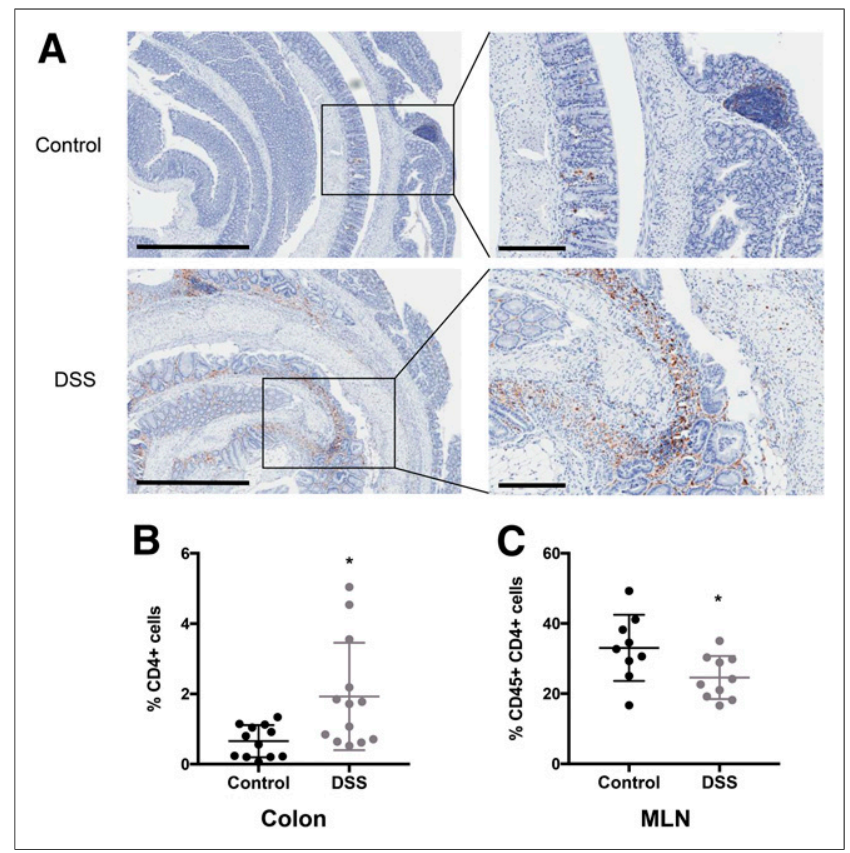

FIGURE 2. Characterization of CD4 cells in colons and MLNs of DSStreated mice. (A) Representative images of antimouse CD4 immunohistochemical staining in colons from control (top) and DSS-treated (bottom) mice. Left panels: $\times 4$ magnification; scale bar, $500 \mu \mathrm{m}$. Right panels: $\times 10$ magnification; scale bar, $200 \mu \mathrm{m}$. (B) Number of cells staining positively for CD4 from entire colon section divided by total number of cells. Two sections of colon, $100 \mu \mathrm{m}$ apart, were analyzed per mouse, with each point representing average percentage of CD4+ T cells in colon from 1 mouse (control, $n=12$ mice; DSS, $n=13$ ). (C) Flow cytometric analysis of CD45+ CD4 + cells from MLNs (control, $n=9$; DSS, $n=10$ ) of control and DSS-treated mice. ${ }^{*} P<0.05$. 


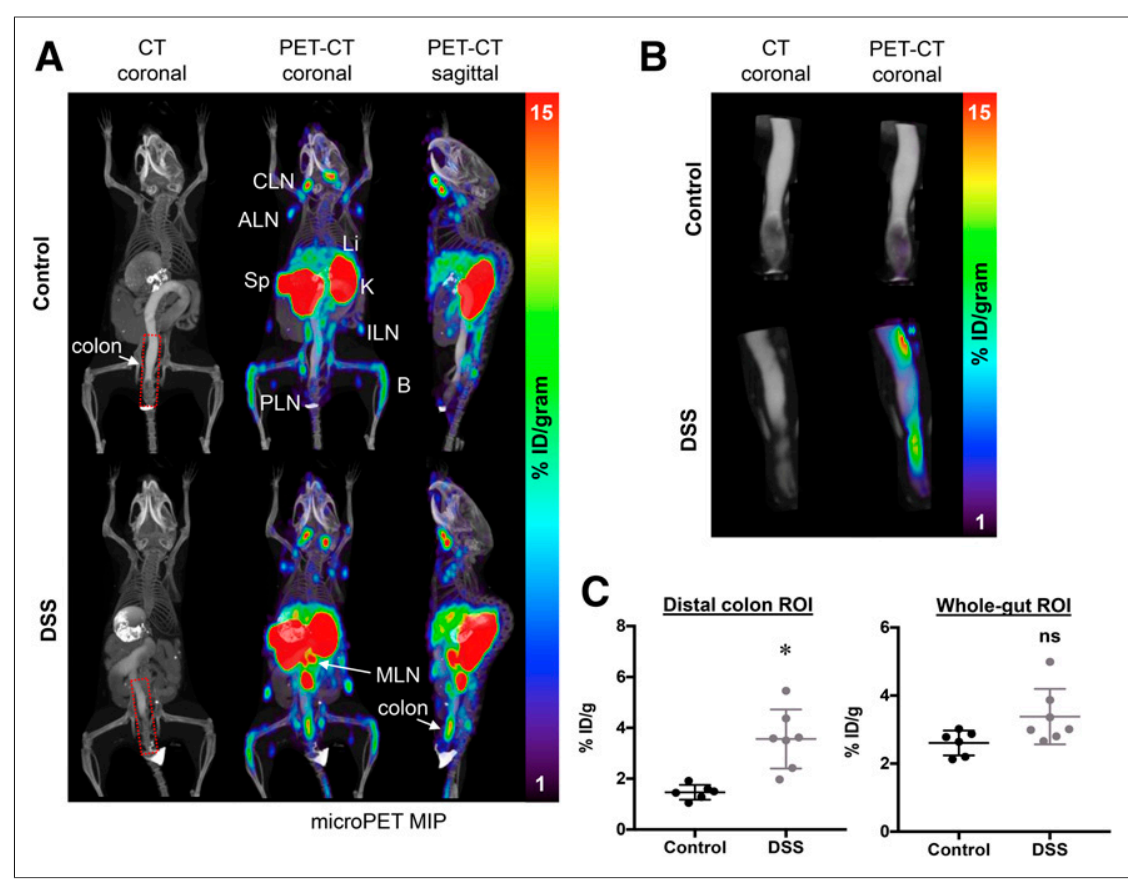

FIGURE 3. Anti-CD4 immuno-PET of control and DSS-treated mice. (A) Representative CT (coronal) and PET/CT (coronal and sagittal) images of control (top) and DSS-treated (bottom) mice acquired with $2 \mu \mathrm{g}(0.34 \mathrm{MBq})$ of $89 \mathrm{Zr}$-malDFO-GK1.5 cDb $(n=8)$. Images were acquired $20 \mathrm{~h}$ after injection of probe and are displayed as $25-\mathrm{mm}$ maximum-intensity projections (MIPs). Organs of interest are axillary LN (ALN), bone (B), cervical LN (CLN), inguinal LN (ILN), kidney (K), liver (Li), popliteal LN (PLN), mesenteric LN (MLN), and spleen (Sp). Distal colon ROI is indicated by red dotted line. (B) Representative CT and PET/CT images of ROI drawn around distal colon from control (top) and DSS-treated (bottom) mice. (C) Quantitative analysis of ROIs drawn around distal colon or small and large intestines, using 3-dimensional isocontour ROI function of AMIDE (control, $n=6$; DSS, $n=7$ ). ${ }^{\star} P<0.005$.

${ }^{89} \mathrm{Zr}$-maleimide-deferoxamine (malDFO)-GK1.5 cDb (2 $\mu \mathrm{g} ; 0.38$ $\mathrm{MBq}$ ) was injected intravenously. One hour before imaging, $100 \mu \mathrm{L}$ of Gastrografin (diatrizoate meglumine and diatrizoate sodium solution; Bracco) were administered orally for CT contrast of the upper gastrointestinal tract. Immediately before imaging, the mice were anesthetized with $2 \%$ isoflurane, and $100 \mu \mathrm{L}$ of Gastrografin were administered intrarectally for large-intestine contrast. PET and CT scans were acquired $20 \mathrm{~h}$ after injection as previously described (18). CT scans were acquired on the CrumpCAT, a fast micro-CT prototype developed at our institution (20). Ex vivo PET/CT scans of harvested colons, ceca, and MLNs were acquired using the same parameters as above. PET/CT images were viewed using AMIDE (http://amide.sourceforge.net). Biodistribution analysis was conducted as previously described (18). Additionally, colons and ceca were fixed and processed for histopathologic and immunohistochemical analysis.

For quantitative analysis of intestinal uptake, first the skeleton was manually removed from the CT image using several regions of interest (ROIs). A 3-dimensional isocontour ROI around the contrast-enhanced small and large intestines (gut ROI) was created. After gut ROI quantification, a cylindric ROI was drawn around a representative colon, from the anus to just below the kidneys, and used on every mouse to reproducibly crop out the same amount of anatomy. A 3-dimensional isocontour ROI was then drawn on the remaining distal colon and quantified.

\section{Flow Cytometry}

Lymphocytes in MLNs and spleens were analyzed by flow cytometry. Organ harvesting and preparation were performed as described previously (18).

\section{Statistical Analysis}

Prism software (GraphPad) was used to perform 2-tailed Student $t$ tests. A $P$ value of less than 0.05 was considered to be statistically significant. For $t$ tests on organs from the ex vivo biodistribution study, the HolmSidak correction for multiple comparisons was applied. Values are reported as mean \pm SD unless indicated otherwise.

\section{RESULTS}

\section{DSS-Induced Colitis Results in Gross Anatomic Changes}

Mice treated with 4\% DSS for $5 \mathrm{~d}$ (Fig. 1A) showed weight loss beginning on day 6. On day 12 , the weight of DSS-treated mice was significantly reduced to $87 \% \pm$ $0.06 \%$ of initial weight, whereas control mice did not display any weight loss $(P<$ 0.0001; $n=8$ ) (Fig. 1B). DSS-treated mice also had significantly higher scores on the disease activity index than control mice as a result of increased weight loss, loose stool, and fecal bleeding (Supplemental Fig. 1A; supplemental materials are available at http:// jnm.snmjournals.org).

Colons from DSS-treated mice were significantly shorter $(P<0.05)$ (Fig. $1 C)$ and heavier (data not shown) than control colons. The colon weight-to-length ratio was significantly higher in colitic mice $(P<0.001)$ (Fig. 1D). Individual MLNs from DSS-treated mice weighed more on average $(6.1 \pm 3.0 \mathrm{mg}$; range, $1.4-11 \mathrm{mg})$ than MLNs from control mice $(2.1 \pm 1.1 \mathrm{mg}$; range, $0.4-4.4 \mathrm{mg} ; P<0.0001 ; n=8$ mice) (Fig. 1E); furthermore, average MLN cellularity was higher in DSS mice $(n=4$ mice; Supplemental Fig. 2).

Histopathologic analysis showed that colons from DSS-treated mice had epithelial cell loss, crypt destruction, and infiltration of inflammatory cells into the mucosa (Supplemental Figs. 1B and 1C). These signs were severe but localized in most mice. DSStreated mice also had significantly more lymphoid aggregates throughout the colon (Supplemental Fig. 1D).

\section{Changes in CD4+ T Cells in DSS-Treated Mice}

Anti-CD4 immunohistochemical staining showed increased infiltration of CD4 $+\mathrm{T}$ cells into the colons of DSS-treated mice (Fig. 2A). CD4 + cells were on average $0.66 \% \pm 0.46 \%$ of the total cells in each section of colon in control mice, whereas DSStreated mice had an average of $1.9 \% \pm 1.5 \% \mathrm{CD} 4+$ cells $(P<$ 0.05; control $n=12$, DSS $n=13$ ) (Fig. 2B). Ceca from colitic mice did not show a significant increase in the percentage of CD4 + T cells (Supplemental Fig. 3A). Changes in CD4+ T cells in spleens and MLNs were evaluated by flow cytometry. The percentage of CD45+ CD4+ cells in the spleen increased slightly in DSS mice, though not significantly (control, 20.6\% \pm 4.4\%; DSS, $23.7 \% \pm 4.1 \% ; n=11$ mice) (Supplemental Fig. 3B). The percentage of CD45+ CD4+ cells in MLNs of DSS mice decreased significantly (control, $33.0 \% \pm 9.4 \%$; DSS, $24.6 \% \pm 6.1 \% ; P<0.05$; control $n=9$ mice; DSS $n=10$ mice) (Fig. 2C). 


\section{Immuno-PET Imaging of CD4 in Colitic Mice}

GK1.5 cDb was site-specifically conjugated to malDFO and radiolabeled with ${ }^{89} \mathrm{Zr}$. Radiolabeling efficiency was more than $99 \%(n=3)$. Specific activity was $0.19 \pm 0.023 \mathrm{MBq} / \mu \mathrm{g}$ $\left(9.9 \times 10^{5} \pm 1.2 \times 10^{5} \mathrm{MBq} / \mathrm{mmol}\right)$, and radiochemical purity was more than $99 \%(n=3) .{ }^{89} \mathrm{Zr}$-malDFO-GK1.5 cDb $(2 \mu \mathrm{g}$; $0.38 \mathrm{MBq}$ ) was injected into groups of mice (2 experiments of 4 mice per group). PET imaging showed tracer uptake in spleens and lymph nodes, consistent with previous results of studies of untreated mice (Fig. 3A) (18). PET scans of colitic mice demonstrated increased signal from MLNs. The colons of colitic mice also showed uptake of ${ }^{89} \mathrm{Zr}$-malDFO-GK1.5 $\mathrm{cDb}$, whereas the control mice showed little to no uptake in the colon (Fig. 3A). Oral and intrarectal instillation of CT contrast agent delineated the intestine clearly on the CT scan (Supplemental Figs. 4A and 4B) and enabled 3-dimensional isocontour ROIs of the complete small and large intestines (gut ROI) and of the distal colon only (colon ROI) to be created on the basis of intensity of CT signal after manual removal of the skeleton (Supplemental Fig. 4C; Fig. 3B). DSS mice had significantly increased uptake in the colon ROI $(P<0.005)$ but not the gut ROI compared with control mice $(P=0.548)$ (Fig. 3C); spillover signal from the kidneys into the ROI may have masked actual differences.

Ex vivo scans confirmed the results of the in vivo scans, showing visibly increased uptake in colons, ceca, and MLNs from DSS mice (Fig. 4). Biodistribution studies showed that total activity was

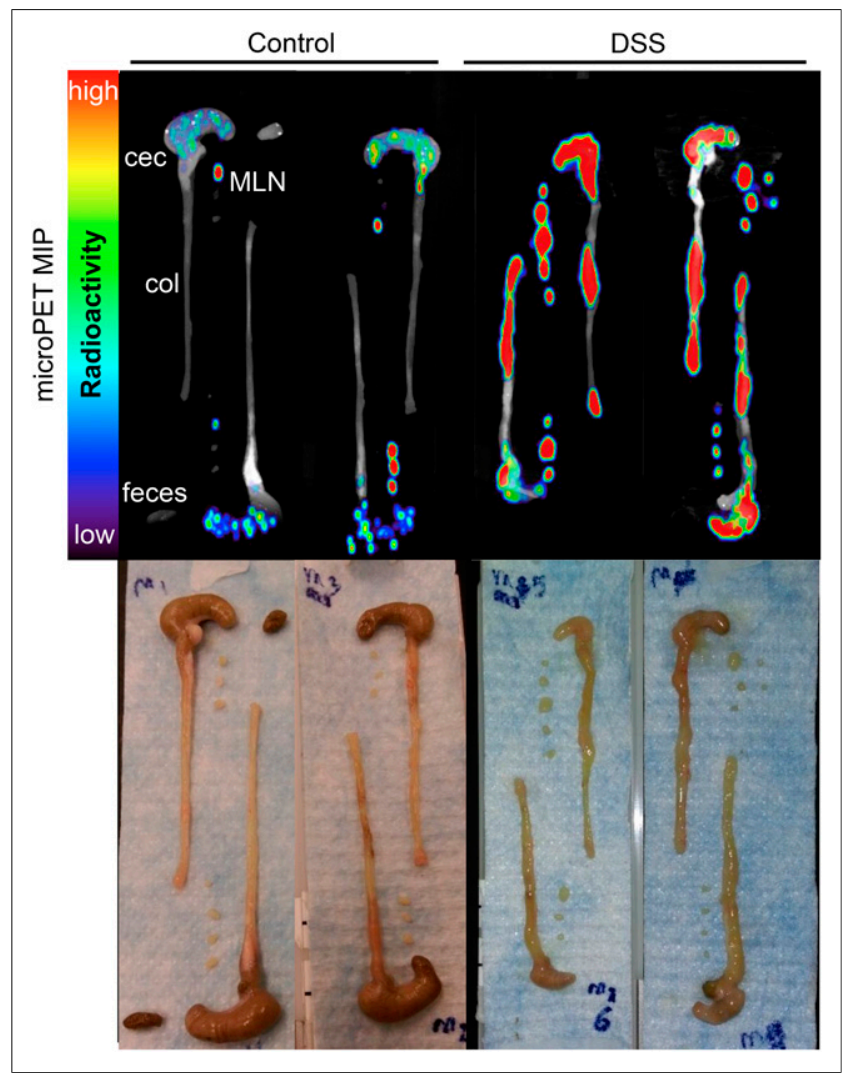

FIGURE 4. Ex vivo immuno-PET images of colons, ceca, and MLNs. After in vivo PET/CT scan mice were euthanized, colons, ceca, and MLNs were dissected and laid out for ex vivo PET/CT maximum-intensity projections (MIPs) ( $n=8$ mice per group). Representative images are shown, displayed as 12-mm maximum-intensity projections. higher in DSS-treated colons, ceca, and MLNs (3.1-fold, 3.9-fold, and 3.0-fold, respectively) (Fig. 5A; Table 1). Comparing concentration of activity (percentage injected dose per organ $[\% \mathrm{ID} / \mathrm{g}]$ ) in organs known to be affected by DSS-induced colitis (colon, cecum, and MLN) did not result in significant changes in uptake (Fig. 5B; Supplemental Table 1).

\section{DISCUSSION}

Aberrant activation and infiltration of CD4+ $\mathrm{T}$ cells is a hallmark of IBD in humans and in numerous mouse models of IBD. The contribution of CD4+ T cells to DSS colitis is still controversial. Although the DSS model does not require $\mathrm{T}$ cells for induction of colitis $(4,5)$, they still infiltrate into the colon (6), and $\mathrm{CD} 4+\mathrm{T}$ cells appear to play a role in the chronic stage of colitis $(21,22)$. Adoptive transfer of DSS-primed CD4+ T cells into recipient mice treated with DSS resulted in exaggerated colitis (23). Conversely, athymic CD-1 nu/nu mice treated with DSS developed more severe clinical signs of colitis than normal mice, suggesting that $\mathrm{T}$ cells may be protective in DSS colitis (5). Molecular imaging could be of use to further investigate how CD4+ $\mathrm{T}$ cells affect the induction and progression of DSS colitis.

The DSS model and resulting changes in CD4 $+\mathrm{T}$ cells were validated using quantitative immunohistochemistry and flow cytometry. Radiolabeled anti-CD4 GK1.5 cDb was used to see if the presence of increased CD4 $+\mathrm{T}$ cells could be visualized and quantified with immuno-PET. ${ }^{89} \mathrm{Zr}$-malDFO-GK1.5 cDb successfully detected CD4 $+\mathrm{T}$ cells in the colons and MLNs of colitic mice, and quantification of the distal colon region with ROI analysis showed that uptake significantly increased in colitic mice. This result is consistent with other studies, which report that the most severe damage in the DSS model occurs in that region $(4,24)$. Quantifying uptake in the gut ROI, which included both the small and the large intestines, was challenging because the probe cleared through the kidneys, resulting in signal spillover into the anterior region of the ROI; differences in the gut ROIs were not significant between control and DSS-treated mice.

Significantly increased uptake of ${ }^{89} \mathrm{Zr}$-malDFO-GK1.5 cDb in the distal colon ROI, as well as in MLNs, was supported by the ex vivo organ scans. Interestingly, uptake was also increased in the ceca of colitic mice, although we did not observe increased CD4+ $\mathrm{T}$ cells by immunohistochemistry in colitic ceca; this may be due at least in part to enhanced permeability of the cecum due to DSSinduced inflammation. Quantification of uptake via ex vivo biodistribution analysis confirmed a significant increase in \% ID per organ for colons, MLNs, and ceca of DSS-treated mice; however, the concentration of activity in these organs (presented as \% ID/g) was not significantly higher in DSS mice. In colons of DSS mice, this may be explained by the finding that they were heavier than control colons. In DSS-treated MLNs, although the percentage of CD4 $+\mathrm{T}$ cells decreased, MLNs were significantly enlarged, which suggests that both CD4 $+\mathrm{T}$ cells and other types of immune cells were present in higher total numbers, resulting in increased size but not increased concentration.

In in situ and ex vivo immuno-PET images, colitic MLNs appear to have a higher \%ID/g than control MLNs, but ex vivo biodistribution analysis indicates that they have the same $\% \mathrm{ID} / \mathrm{g}$. This finding is explained by the partial-volume effect, in which the concentration of activity in organs near the resolution of the PET scanner is underestimated (17). Colitic MLNs were dramatically enlarged and more easily detected by immuno-PET than the tiny 


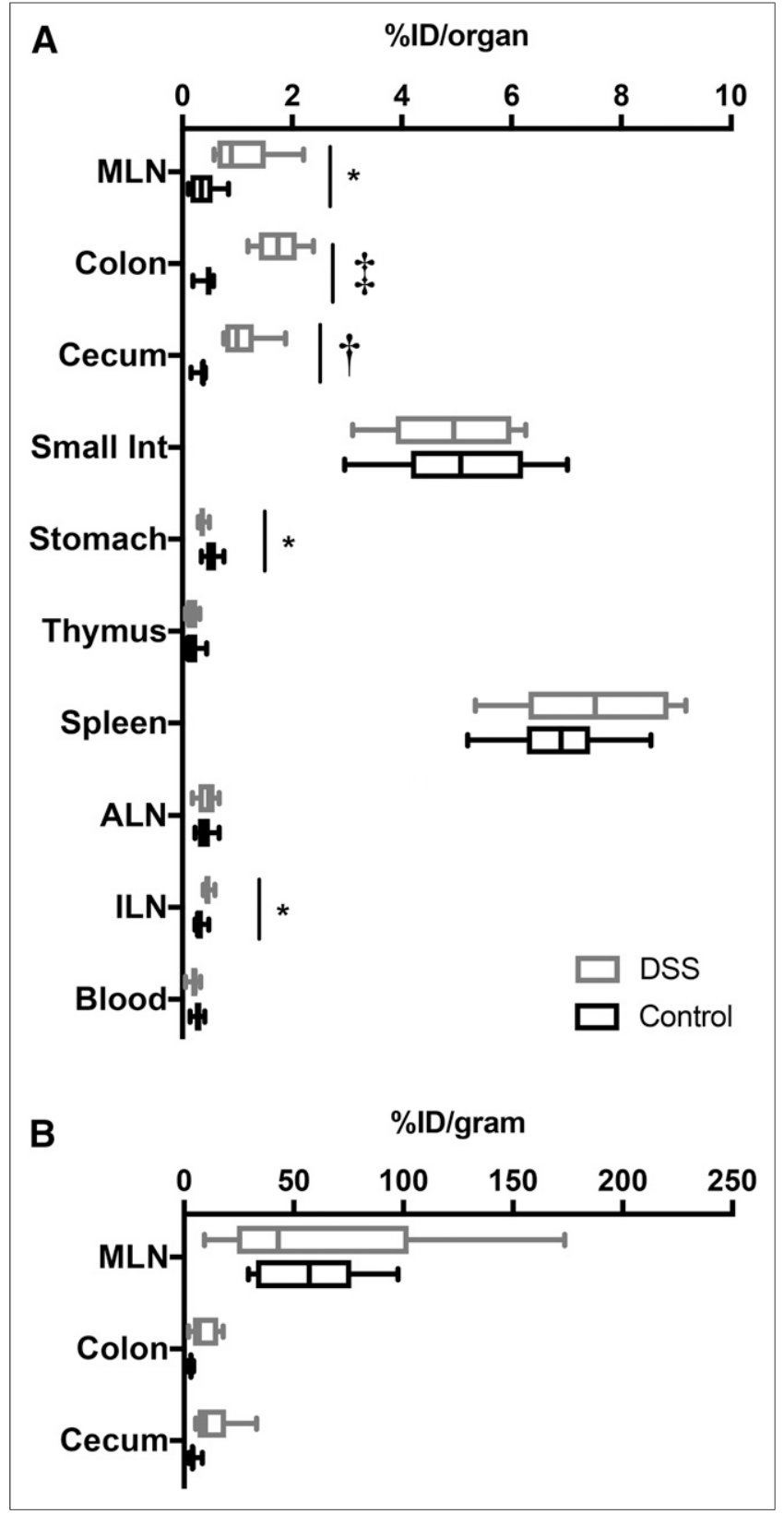

FIGURE 5. Ex vivo biodistribution of organs from control and DSStreated mice. After PET scan, mice were euthanized, and blood and organs were removed, weighed, and counted. Decay-corrected values for uptake are displayed. (A) \%ID/organ for lymphoid and gastrointestinal organs. ${ }^{*} P<0.05$. ${ }^{\dagger} P<0.005$. ${ }^{\ddagger} P<0.0001$. (B) $\%$ ID/g for organs of interest: colon, cecum, and MLN ( $n=8)$. Box shows $25 \%$ quartile, median, and $75 \%$ quartiles. Whiskers indicate minimum and maximum values. $A L N=$ axillary $L N ; I L N=$ inguinal $L N$.

control MLNs. The partial-volume effect, especially in the context of murine lymph nodes, has been observed and discussed in previous work from our laboratory (17). The observation of signal from enlarged MLNs in colitic mice demonstrates the importance of MLNs and associated lymphatics in the development of DSS colitis and raises the possibility of the prognostic value of inflammatory staging sites such as MLNs, though more work is needed to fully understand the timing and cellular dynamics of MLN enlargement.
These results confirm and expand on the work of Kanwar et al., who used ${ }^{111}$ In-labeled intact anti-CD4 antibody for SPECT imaging of CD4+ $\mathrm{T}$ cells in DSS mice; in that work, a protein dose of $350 \mu \mathrm{g}$ was used (14). We previously explored protein doses and found that amounts as low as $2 \mu \mathrm{g}$ of GK1.5 cDb enabled imaging of $\mathrm{CD} 4+\mathrm{T}$ cells in the lymph nodes and spleen with minimal biologic effects (18). Here, $2 \mu \mathrm{g}$ of GK1.5 cDb were sufficient to image CD4+ T cells in the DSS model. Furthermore, Kanwar et al. drew an ROI that encompassed the entire lower abdomen and referred to this as "colon uptake" but did not discuss signal from CD4 + T cells in the MLN (14), which were likely included in the ROI. Therefore, while whole-abdomen ROIs may have prognostic value in IBD, they must be interpreted with consideration of the presence of MLNs and associated lymphatic tissue.

Although GK1.5 cDb enables visualization of total CD4+ cells in the gut, it does not provide information about CD4+ T-cell subsets such as Th1, Th2, Th17, or regulatory $\mathrm{T}$ cells, which is disadvantageous given the different pro- and antiinflammatory roles of each of these subsets. However, GK1.5 cDb can be used to identify sites of CD4+ T-cell accumulation, followed by detailed analysis with traditional methods such as biopsy to determine the subsets present as needed. Further studies using GK1.5 cDb in

TABLE 1

Ex Vivo Biodistribution

\begin{tabular}{|c|c|c|c|c|c|c|}
\hline \multirow[b]{2}{*}{ Site } & \multicolumn{3}{|c|}{ Control } & \multicolumn{3}{|c|}{ DSS } \\
\hline & Mean & SD & $n$ & Mean & SD & $n$ \\
\hline MLN & 0.37 & 0.25 & 8 & $1.09^{\star}$ & 0.58 & 8 \\
\hline Colon & 0.45 & 0.12 & 8 & $1.75^{\ddagger}$ & 0.40 & 8 \\
\hline Cecum & 0.35 & 0.09 & 8 & $1.09^{\dagger}$ & 0.38 & 8 \\
\hline Small int. & 5.12 & 1.33 & 8 & 4.87 & 1.13 & 8 \\
\hline Stomach & 0.53 & 0.13 & 8 & $0.37^{\star}$ & 0.07 & 8 \\
\hline Thymus & 0.17 & 0.14 & 8 & 0.16 & 0.11 & 8 \\
\hline Spleen & 6.89 & 0.98 & 8 & 7.56 & 1.38 & 8 \\
\hline ALN & 0.41 & 0.14 & 8 & 0.45 & 0.17 & 8 \\
\hline ILN & 0.31 & 0.09 & 8 & $0.46^{\star}$ & 0.07 & 8 \\
\hline Blood & 0.28 & 0.08 & 8 & 0.22 & 0.08 & 8 \\
\hline Liver & 9.84 & 2.64 & 8 & 13.37 & 1.01 & 8 \\
\hline Kidney & 23.84 & 6.26 & 8 & 27.30 & 3.68 & 8 \\
\hline Heart & 0.15 & 0.04 & 8 & 0.21 & 0.07 & 8 \\
\hline Lung & 0.38 & 0.26 & 8 & 0.36 & 0.03 & 8 \\
\hline Muscle & 0.04 & 0.01 & 8 & 0.05 & 0.01 & 8 \\
\hline Femur & 0.69 & 0.17 & 8 & 0.49 & 0.10 & 8 \\
\hline Tail & 4.19 & 3.63 & 8 & 3.25 & 0.72 & 8 \\
\hline Carcass & 19.53 & 3.24 & 8 & 16.64 & 0.96 & 8 \\
\hline \multicolumn{7}{|c|}{$\begin{array}{l}{ }^{\star} P<0.05 . \\
{ }^{\dagger} P<0.005 . \\
{ }^{\ddagger} P<0.0001 . \\
\text { Int. = intestine; ALN = axillary LN; ILN = inguinal LN. } \\
\text { Data are total activity (\%ID) present in blood and organs from } \\
\text { SSS-treated mice compared with control mice } 26 \mathrm{~h} \text { after injection } \\
\text { f }{ }^{89} \mathrm{Zr} \text {-malDFO-GK } 1.5 \mathrm{cDb} \text {. }\end{array}$} \\
\hline
\end{tabular}


mouse models of IBD could include imaging of CD4 in a T-celldependent model or imaging at several time points during the development of colitis to investigate the course of T-cell infiltration into the intestines and MLNs.

$\mathrm{CD} 4+\mathrm{T}$ cells play a key role in human IBD, and identifying them throughout the bowel could be useful to monitor disease severity and predict treatment success. Imaging is less intrusive than colonoscopies, which are uncomfortable for patients and carry a risk of bowel perforation; furthermore, inflammation can vary depending on the position within the bowel. Anti-CD4 immuno-PET could provide an overview of inflammatory sites throughout the intestines. Other immune targets, including granulocytes (24), E-selectin (25), and CXCL8 (12), have been imaged in IBD patients. Aarntzen et al. suggest that anti-CXCL8 imaging could be a tool for making decisions about immune-modulating treatment (12); a similar application could apply for anti-CD4 imaging.

\section{CONCLUSION}

Anti-CD4 immuno-PET with GK1.5 cDb enabled detection of CD4 $+\mathrm{T}$ cells in inflamed mouse colons and in enlarged MLNs and should provide a useful tool for investigating CD4 $+\mathrm{T}$ cells in murine models of IBD and other diseases with a CD4 inflammatory component.

\section{DISCLOSURE}

Histological analysis was provided by the UCLA Imaging and Stem Cell Core at the CURE Digestive Diseases Research Center (NIH P30DK41301). Flow cytometry was performed in the UCLA Jonsson Comprehensive Cancer Center (JCCC) and the Center for AIDS Research Flow Cytometry Core Facility (NIH P30CA016042 and 5P30AI028697). Small-animal PET scans were performed in the Preclinical Imaging Technology Core at the Crump Institute for Molecular Imaging at UCLA, which is funded by the UCLA JCCC and National Institutes of Health (NIH P30CA016042). Amanda C. Freise was supported by the Dissertation Year Fellowship from UCLA. Richard Tavaré was funded by NIH award R21AI114255 and the UCLA Scholars in Oncologic Molecular Imaging training program (NIH R25T098010). Anna M. Wu is a member of the JCCC and is a stockholder in and consultant to ImaginAb, Inc. No other potential conflict of interest relevant to this article was reported.

\section{ACKNOWLEDGMENTS}

We thank Kha Huynh, Alec Estrada, Waldemar Ladno, Theresa Falls, Jason Lee, Richard Taschereau, and Clara Magyar for technical assistance.

\section{REFERENCES}

1. Brandtzaeg P, Kiyono H, Pabst R, Russell MW. Terminology: nomenclature of mucosa-associated lymphoid tissue. Mucosal Immunol. 2008;1:31-37.

2. Geremia A, Biancheri P, Allan P, Corazza GR, Di Sabatino A. Innate and adaptive immunity in inflammatory bowel disease. Autoimmun Rev. 2014;13:3-10.

3. Wirtz S, Neurath MF. Mouse models of inflammatory bowel disease. Adv Drug Deliv Rev. 2007;59:1073-1083.

4. Dieleman LA, Ridwan BU, Tennyson GS, Beagley KW, Bucy RP, Elson CO. Dextran sulfate sodium-induced colitis occurs in severe combined immunodeficient mice. Gastroenterology. 1994;107:1643-1652.
5. Axelsson LG, Landström E, Goldschmidt TJ, Grönberg A, Bylund-Fellenius AC. Dextran sulfate sodium (DSS) induced experimental colitis in immunodeficient mice: effects in CD4+-cell depleted, athymic and NK-cell depleted SCID mice. Inflamm Res. 1996;45:181-191.

6. Hall LJ, Faivre E, Quinlan A, Shanahan F, Nally K, Melgar S. Induction and activation of adaptive immune populations during acute and chronic phases of a murine model of experimental colitis. Dig Dis Sci. 2011;56:79-89.

7. Hindryckx P, Staelens S, Devisscher L, et al. Longitudinal quantification of inflammation in the murine dextran sodium sulfate-induced colitis model using microPET/CT. Inflamm Bowel Dis. 2011;17:2058-2064.

8. Bettenworth D, Reuter S, Hermann S, et al. Translational ${ }^{18}$ F-FDG PET/CT imaging to monitor lesion activity in intestinal inflammation. J Nucl Med. 2013;54: 748-755.

9. Brewer S, McPherson M, Fujiwara D, et al. Molecular imaging of murine intestinal inflammation with 2-deoxy-2-[18$\left.{ }^{18} \mathrm{~F}\right]$ fluoro-D-glucose and positron emission tomography. Gastroenterology. 2008;135:744-755.

10. Treglia G, Quartuccio N, Sadeghi R, et al. Diagnostic performance of fluorine18-fluorodeoxyglucose positron emission tomography in patients with chronic inflammatory bowel disease: a systematic review and a meta-analysis. J Crohns Colitis. 2013;7:345-354.

11. Glaudemans AWJM, de Vries EFJ, Galli F, Dierckx RAJO, Slart RHJA, Signore A. The use of ${ }^{18} \mathrm{~F}$-FDG-PET/CT for diagnosis and treatment monitoring of inflammatory and infectious diseases. Clin Dev Immunol. 2013;2013: 623036.

12. Aarntzen EHJG, Hermsen R, Drenth JPH, Boerman OC, Oyen WJG. ${ }^{99 m} \mathrm{Tc}-$ CXCL8 SPECT to monitor disease activity in inflammatory bowel disease. J Nucl Med. 2016;57:398-403.

13. Freise $\mathrm{AC}, \mathrm{Wu} \mathrm{AM}$. In vivo imaging with antibodies and engineered fragments. Mol Immunol. 2015;67:142-152.

14. Kanwar B, Gao DW, Hwang AB, et al. In vivo imaging of mucosal CD4 + T cells using single photon emission computed tomography in a murine model of colitis. J Immunol Methods. 2008;329:21-30.

15. Dearling JLJ, Park EJ, Dunning P, et al. Detection of intestinal inflammation by microPET imaging using a ${ }^{64} \mathrm{Cu}$-labeled anti-beta7 integrin antibody. Inflamm Bowel Dis. 2010;16:1458-1466.

16. Dearling JLJ, Daka A, Veiga N, Peer D, Packard AB. Colitis immunoPET: defining target cell populations and optimizing pharmacokinetics. Inflamm Bowel Dis. 2016;22:529-538.

17. Tavaré R, McCracken MN, Zettlitz KA, Salazar FB, Witte ON, Wu AM. ImmunoPET of murine $\mathrm{T}$ cell reconstitution post-adoptive stem cell transplant using anti-CD4 and anti-CD8 cys-diabodies. J Nucl Med. 2015;56: 1258-1264.

18. Freise AC, Zettlitz KA, Salazar FB, Lu X, Tavaré R, Wu AM. ImmunoPET imaging of murine CD4 $+\mathrm{T}$ cells using anti-CD4 cys-diabody: effects of protein dose on T cell function and imaging. Mol Imaging Biol. 2017;19: 599-609.

19. Sha T, Igaki K, Yamasaki M, Watanabe T, Tsuchimori N. Establishment and validation of a new semi-chronic dextran sulfate sodium-induced model of colitis in mice. Int Immunopharmacol. 2013;15:23-29.

20. Taschereau R, Vu NT, Chatziioannou AF. Calibration and data standardization of a prototype bench-top preclinical CT. In: Nuclear Science Symposium and Medical Imaging Conference (NSS/MIC). Piscataway, NJ: IEEE; 2015: 15853923.

21. Dieleman LA, Palmen MJ, Akol H, et al. Chronic experimental colitis induced by dextran sulphate sodium (DSS) is characterized by Th1 and Th2 cytokines. Clin Exp Immunol. 1998;114:385-391.

22. Melgar S, Karlsson A, Michaëlsson E. Acute colitis induced by dextran sulfate sodium progresses to chronicity in C57BL/6 but not in BALB/c mice: correlation between symptoms and inflammation. Am J Physiol Gastrointest Liver Physiol. 2005;288:G1328-G1338.

23. Shintani N, Nakajima $\mathrm{T}$, Okamoto $\mathrm{T}$, Kondo $\mathrm{T}$, Nakamura $\mathrm{N}$, Mayumi $\mathrm{T}$. Involvement of CD4+ $\mathrm{T}$ cells in the development of dextran sulfate sodiuminduced experimental colitis and suppressive effect of IgG on their action. Gen Pharmacol. 1998;31:477-481.

24. Bruno I, Martelossi S, Geatti O, et al. Antigranulocyte monoclonal antibody immunoscintigraphy in inflammatory bowel disease in children and young adolescents. Acta Paediatr. 2002;91:1050-1055.

25. Bhatti M, Chapman P, Peters M, Haskard D, Hodgson HJF. Visualising E-selectin in the detection and evaluation of inflammatory bowel disease. Gut. 1998;43: 40-47. 